\title{
Technical note: Development and application of KASP assays for rapid screening of 8 genetic defects in Holstein cattle
}

\author{
Yi Zhang, ${ }^{1 *}$ † Dong Liang, ${ }^{1} \dagger$ Hetian Huang, ${ }^{1}$ Zhancheng Yang, ${ }^{1}$ Yachun Wang, ${ }^{1}$ Ying Yu, ${ }^{1}$ Lin Liu, ${ }^{2}$ \\ Shengli Zhang, ${ }^{1}$ Jianlin Han, ${ }^{3,4}$ and Wei Xiao ${ }^{5 *}$ \\ ${ }^{1}$ National Engineering Laboratory for Animal Breeding, Key Laboratory of Animal Genetics, Breeding and Reproduction, \\ Ministry of Agriculture and Rural Affairs, College of Animal Science and Technology, China Agricultural University, Beijing 100193, China \\ ${ }^{2}$ Beijing Dairy Cattle Center, Beijing 100192, China \\ ${ }^{3}$ CAAS-ILRI Joint Laboratory on Livestock and Forage Genetic Resources, Institute of Animal Science, \\ Chinese Academy of Agricultural Sciences (CAAS), Beijing 100193, China \\ ${ }^{4}$ International Livestock Research Institute (ILRI), Nairobi 00100, Kenya \\ ${ }^{5}$ Beijing Animal Husbandry Station, Beijing 100107, China
}

\section{ABSTRACT}

Specific DNA mutations underlying several genetic defects associated with embryo loss or reduced calf survivability have been identified in dairy cattle, and a convenient and cost-effective platform is required for their routine screening. We developed Kompetitive allele-specific PCR (KASP) assays for discrimination of the wild-type alleles from the associated defective alleles at each of 8 common genetic defects in Holstein cattle, involving $5 \mathrm{SNP}$ [HH1, HH3, HH4, bovine leukocyte adhesion deficiency (BLAD), and complex vertebral malformation (CVM)] and 3 insertion or deletion mutations [HH5, haplotype for cholesterol deficiency (HCD), and brachyspina (BS)]. A total of 390 cows from a Chinese Holstein herd were genotyped and the carriers identified at 7 of these 8 loci (except HH4), with the highest carrier frequencies found for CVM (10.5\%) and HH1 (10.0\%), followed by HH3 $(2.6 \%)$, BS (2.1\%), HCD (1.3\%), HH5 (0.8\%), and BLAD (0.5\%). Surprisingly, 102 cows $(26.2 \%)$ carried at least 1 of the 7 defective alleles. Our results demonstrate that these KASP assays are simple, rapid, and reliable for the detection of multiple genetic defects. The high carrier frequency of these genetic defects indicates an urgent need for routine molecular testing to eliminate the deleterious alleles from Chinese Holstein cattle.

Key words: allele-specific PCR, genotyping, genetic defect, recessive allele

Received January 22, 2019.

Accepted August 2, 2019.

*Corresponding authors: yizhang@cau.edu.cn and xiao84929056@ 126.com

$\dagger$ These authors contributed equally to this work.

\section{Technical Note}

In dairy cattle, intense selection and use of elite sires have achieved significant genetic improvement of economically important traits. However, they have also led to the accumulation of inbreeding and increasing prevalence of deleterious recessive alleles. In the past decades, several genetic defects, including bovine leukocyte adhesion deficiency (BLAD; Shuster et al., 1992), complex vertebral malformation (CVM; Agerholm et al., 2001), and brachyspina (BS; Charlier et al., 2012) have been reported for Holstein cattle. Additional autosomal recessive lethal defects, such as Holstein haplotypes 1 through5 (HH1 through HH5; VanRaden et al., 2011b; Fritz et al., 2013; Cole et al., 2018) and a specific haplotype for cholesterol deficiency (HCD; Kipp et al., 2015), were detected by identifying, based on genomic SNP data, such haplotypes at high frequencies but with no associated homozygotes. The causative mutations have been identified for BLAD (Shuster et al., 1992), CVM (Thomsen et al., 2006), BS (Charlier et al., 2012), HH1 (Adams et al., 2016), HH3 (Daetwyler et al., 2014; McClure et al., 2014), HH4 (Fritz et al., 2013), HH5 (Schütz et al., 2016), and HCD (Menzi et al., 2016; Schütz et al., 2016). In terms of the mutation types, point mutations are responsible for HH1, HH3, $\mathrm{HH}$, CVM, and BLAD, whereas a large insertion or deletion leads to HH5, HCD, and BS (Table 1). The genomic region responsible for $\mathrm{HH} 2$ was mapped on chromosome 1 from 94,860,836 to 96,553,339 bp (Bos taurus genome assembly UMD3.1), but the causative mutation has yet to be discovered (McClure et al., 2014).

These inherited disorders, all controlled by autosomal recessive alleles, are expressed as a disease phenotype if such alleles are present in a homozygous state. That is, a quarter of the offspring can be affected when both 
Zhang et al.: TECHNICAL NOTE: KASP ASSAYS FOR GENETIC DEFECTS

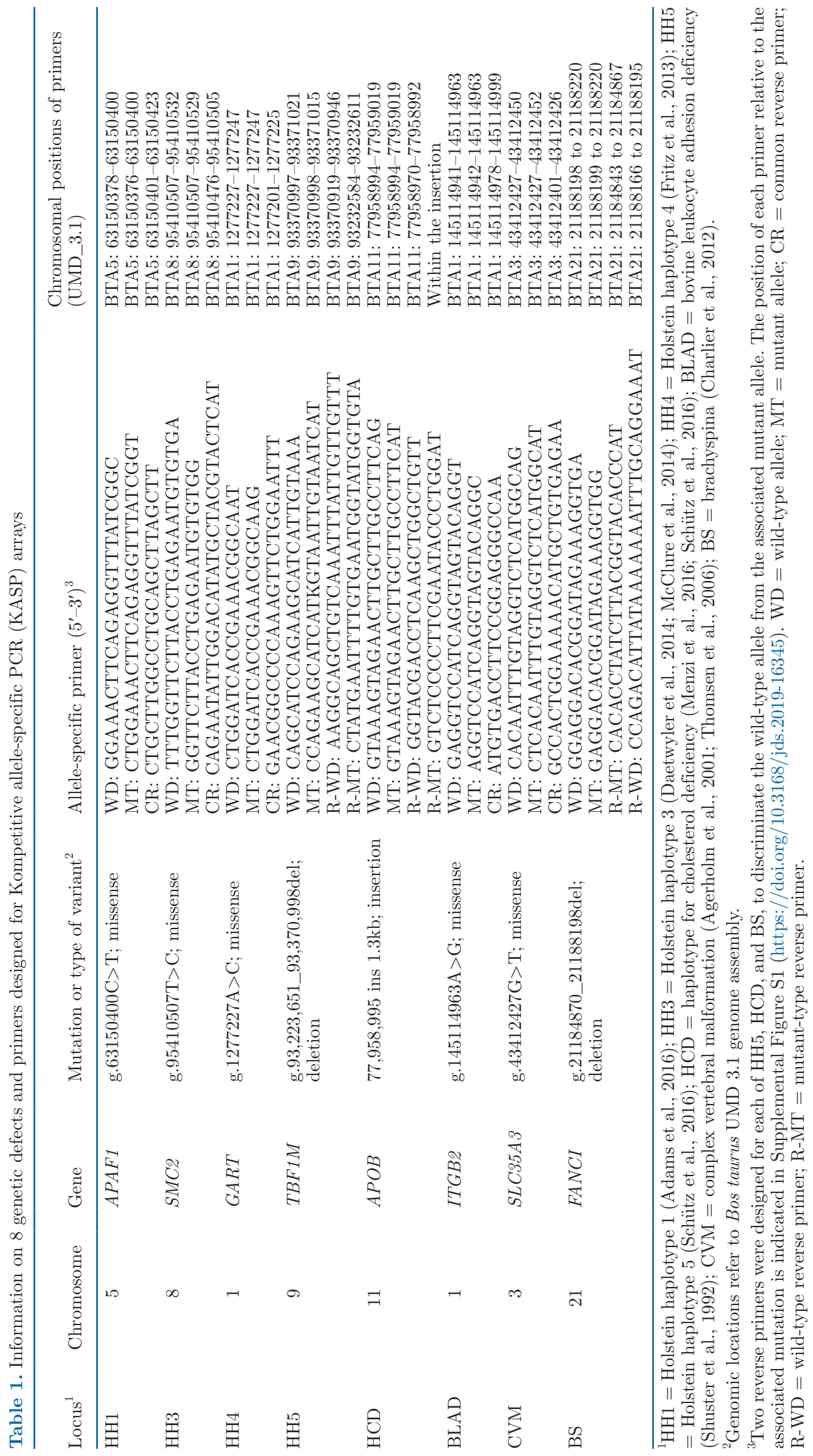


parents are carriers of defective alleles. These genetic defects are associated with early embryonic loss and reduced calf survivability, leading to severe economic losses (Cole et al., 2016; Segelke et al., 2016). Therefore, identification of the carrier animals based on genotyping is a prerequisite for the control and elimination of such defects.

For a defect with known molecular basis, the direct detection of carriers is possible at the DNA level. Different molecular methods, such as PCR-RFLP for HH4 (Fritz et al., 2013), TaqMan for CVM and BLAD (Zhang et al., 2012), and PCR analysis for BS (Charlier et al., 2012; Fang et al., 2013), HCD (Menzi et al., 2016; Schütz et al., 2016), and HH5 (Schütz et al., 2016), have been applied to identify heterozygous individuals. However, the identification of multiple defects requires a unified and flexible assay. The recently developed Kompetitive allele-specific PCR (KASP) has made it possible to efficiently and simultaneously screen different loci (He et al., 2014; Semagn et al., 2014). This is based on an allele-specific PCR followed by bi-allelic scoring of the SNP and insertion or deletion mutations associated with particular loci (He et al., 2014). The aim of this study was to develop an effective and convenient method to accurately diagnose these 8 common genetic defects (HH1, HH3, HH4, HH5, HCD, BLAD, CVM, and BS) in Chinese Holstein cattle.

Sequences of different alleles at these 8 loci were retrieved according to the published literature (Table 1) and then aligned to the cattle reference genome (Bos taurus genome assembly UMD3.1) using the Nucleotide BLAST tool available at the National Center for Biotechnology Information (https://blast.ncbi.nlm.nih .gov/Blast.cgi). Results show that in addition to the published mutations for specific defective alleles, a 9-bp insertion (TGATTACAA) is present immediately after the 138-kb deletion for the HH5 mutant allele (Supplemental Figure S1; https://doi.org/10.3168/jds .2019-16345). Two allele-specific forward primers, one for the wild-type allele and the other for the mutant allele, along with a common reverse primer, were designed for each genotyping assay of the SNP (Table 1, Supplemental Figure S1; https://doi.org/10.3168/ jds.2019-16345), following standard KASP guidelines (https://biosearch-cdn.azureedge.net/assetsv6/KASP -genotyping-chemistry-User-guide.pdf). However, for HH5, HCD, and BS, which are caused by either a large insertion or a large deletion, a second allele-specific reverse primer was designed for each assay to incorporate the mutant sequence (Table 1, Supplemental Figure S1; https://doi.org/10.3168/jds.2019-16345).

A panel of 50 reference samples with known genotypes at these 8 loci were used for validation of the KASP assays developed in this study. The genotypes of these samples have been determined by classical genotyping methods: agarose gel electrophoresis (HH5, HCD, and BS) or direct Sanger sequencing (HH1, HH3, $\mathrm{HH} 4, \mathrm{BLAD}$, and CVM) of the respective PCR products (Supplemental Table S1; https://doi.org/10.3168/ jds.2019-16345). Both wild-type homozygotes and the carriers of mutant alleles for the 8 genetic defects exist in these reference samples (Supplemental Table S2, File S1; https://doi.org/10.3168/jds.2019-16345), which were prepared in-house at the National Engineering Laboratory for Animal Breeding, China Agricultural University (Beijing), and are publicly available for the research community and the dairy industry. The gel electrophoresis results of HH5, HCD, and BS were further verified through Sanger sequencing of the 50 reference samples, using the primers listed in Supplemental Table S1 (https://doi.org/10.3168/jds.2019 -16345). In addition, the alignments of new sequences to the Bos taurus reference genome (UMD3.1) identified 2 SNP (rs132900320, rs133996578) on chromosome 9 at 93,370,986 and 93,371,229 bp, respectively, for the HH5 locus, and a 1-bp deletion on chromosome 11 at $77,959,132 \mathrm{bp}$ for the HCD locus (Supplemental File S1; https://doi.org/10.3168/jds.2019-16345).

Microfluidic-based IMAP platform (CapitalBio Technology, Beijing, China) was employed to conduct PCR amplification. The final reaction was performed in a total volume of $1 \mu \mathrm{L}$, which contained $0.3 \mu \mathrm{L}$ of DNA template $(50 \mathrm{ng} / \mu \mathrm{L}), 0.14 \mu \mathrm{L}$ of primer mix $(12 \mu \mathrm{M}$ each of the 2 allele-specific forward primers and $30 \mu M$ reverse primer), $0.5 \mu \mathrm{L}$ of $2 \times$ universal KASP Master Mix (LGC, Teddington, UK) and $0.06 \mu \mathrm{L}$ of ultrapure water. The PCR thermocycling was performed under the following conditions: initiation at $95^{\circ} \mathrm{C}$ for $15 \mathrm{~min}$; 10 cycles of denaturation at $95^{\circ} \mathrm{C}$ for $20 \mathrm{~s}$; touchdown annealing from $61^{\circ} \mathrm{C}\left(-0.6^{\circ} \mathrm{C} /\right.$ cycle $)$ for $60 \mathrm{~s}$; followed by 26 cycles of denaturation at $95^{\circ} \mathrm{C}$ for $20 \mathrm{~s}$ and annealing at $55^{\circ} \mathrm{C}$ for $60 \mathrm{~s}$; finally, an extension at $37^{\circ} \mathrm{C}$ for $60 \mathrm{~s}$.

An end point fluorescent read of the PCR products was conducted using the LuxScan-10K/D instrument (CapitalBio Technology). Individual genotypes were determined for the 50 reference samples based on the scatter plot of fluorescent signals (Figure 1). Two genotypes, for wild-type homozygote and mutant carriers, were observed for all 8 loci, with individual genotypes matched to all expected genotypes of the 50 reference samples, except for 1 sample of HOLBJ024 at the HH5 locus, which showed an intermediate signal on the plot and was recorded as no call (Figure 1; Supplemental Table S2, https://doi.org/10.3168/jds.2019-16345). Nevertheless, compared with the genotyping result of the 50 reference samples determined by Sanger sequencing, the KASP assays matched $99.75 \%$ of all 

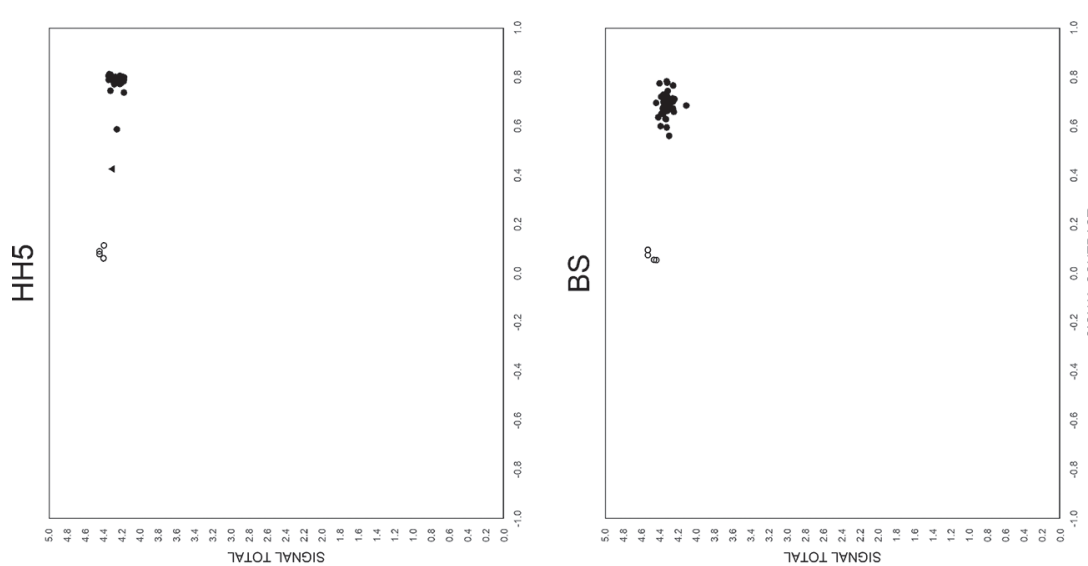

프료

क⿺辶

훙

of

용

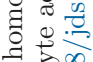

o

可

.

उ.

붕

㻤 11

过定

要造

용
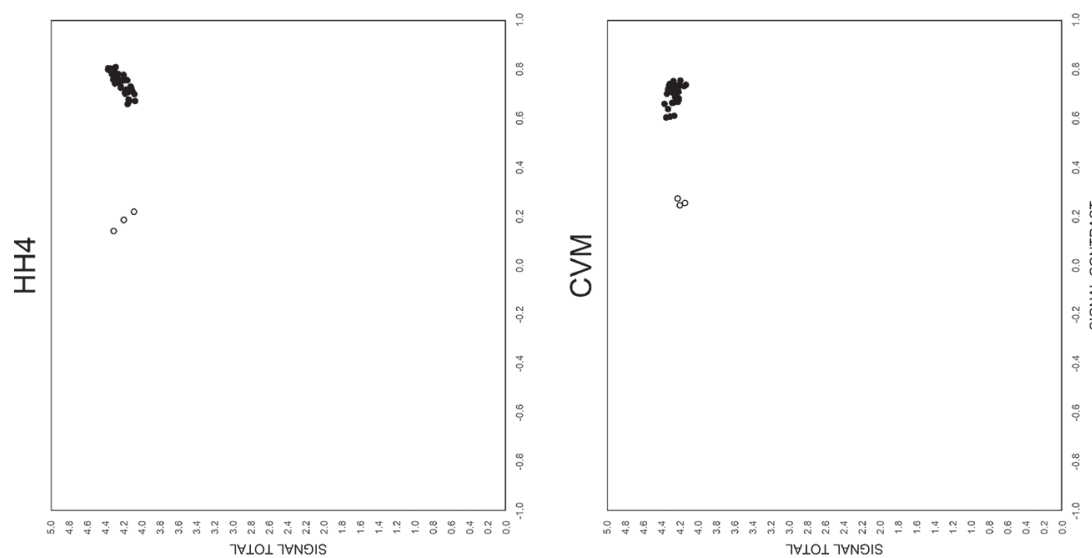

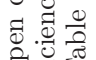

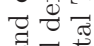

징

궁

की

क्षे

웜

㻤

엉

을

콩

월 워

نी

입 파

的
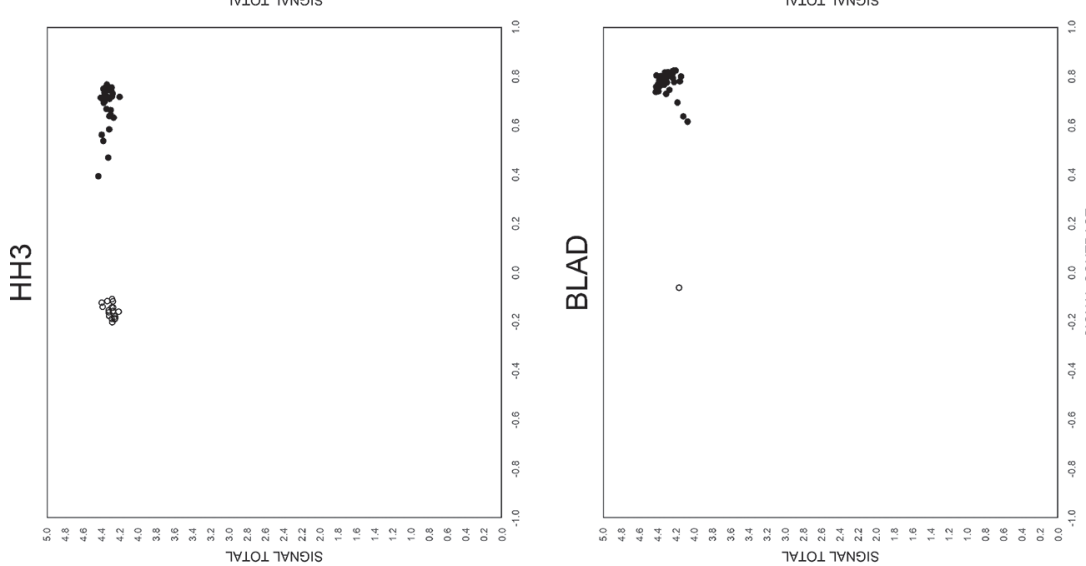

का 군

ज约

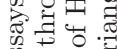

政 $\rightarrow$

两设

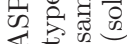

능응

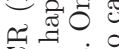

용. 西

낭

o

证

क्षे

造 11

ส

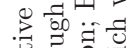
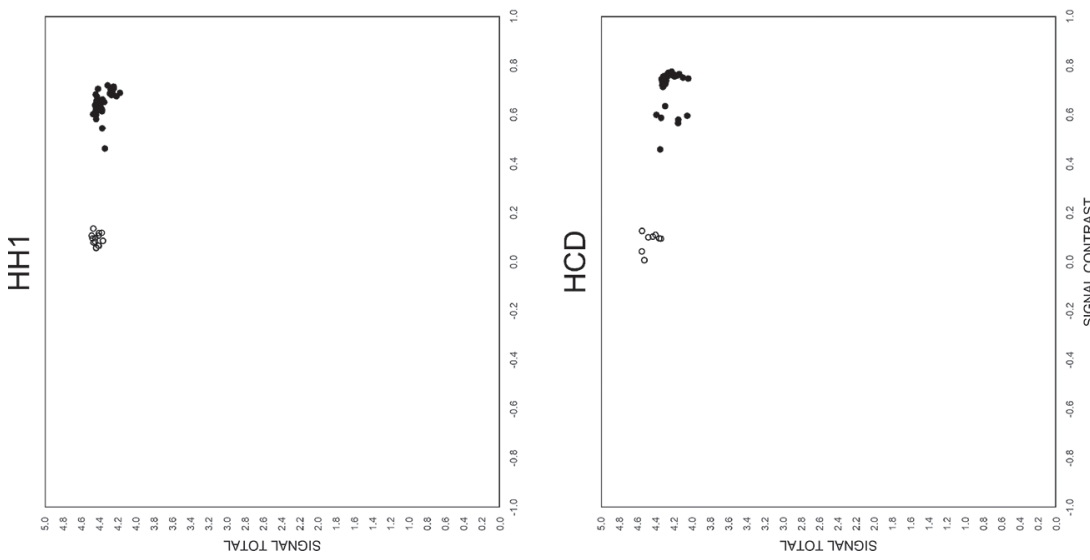

论

:

结啳

$\infty$ 品

业

잉

क क्रे

은

25

.

ฮ웡

क. 11

政表

ํำ

50.0

In 
corresponding genotypes across the 8 loci, suggesting high sensitivity and specificity.

The KASP assays were then applied to screen 390 Chinese Holstein cows, randomly selected from a dairy farm in Beijing, for the prevalence of these 8 genetic defects (Supplemental Figure S2; https://doi.org/10 $.3168 /$ jds.2019-16345). The highest carrier frequencies were observed for CVM (10.5\%) and HH1 (10.0\%), followed by HH3 (2.6\%), BS (2.1\%), HCD (1.3\%), HH5 $(0.8 \%)$, and BLAD (0.5\%). No carrier was found for HH4 (Supplemental Table S3, Figure S2; https://doi .org/10.3168/jds.2019-16345). It was surprising that 102 cows $(26.2 \%)$ carried at least 1 of the 8 defective alleles and 6 cows carried 2 such alleles. These high carrier incidences call for urgent action of routine molecular screening for these known genetic defects, to eliminate them quickly by integrating pedigree data of the affected animals from the ongoing Holstein cattle breeding program in China.

To verify the recessive mutations (Supplemental Table S3, Figure S2; https://doi.org/10.3168/jds.2019 -16345) associated with the genotyping results from KASP assays in the 390 Chinese Holstein cows, Sanger sequencing was performed for PCR products of HH1, HH3, HH4, BLAD, and CVM, and agarose gel electrophoresis was applied for PCR products of $\mathrm{HH} 5$, HCD, and BS, amplified using the primers presented in Supplemental Table S1 (https://doi.org/10.3168/jds .2019-16345). All carriers and 5 randomly selected noncarriers were examined across the 8 defective loci. The results completely matched those obtained via KASP assays, verifying their accuracy for genotyping of these 8 genetic defects (Supplemental Figures S3, S4; https:/ /doi.org/10.3168/jds.2019-16345).

In recent years, several genetic defects associated with yield, longevity, and fertility have been identified in dairy cattle (Cole et al., 2016). The common strategy for screening multiple genetic defects used the haplotype-based method. However, haplotype analysis relies on SNP array genotyping (VanRaden et al., 2011b), which is usually more time-consuming and costly than direct molecular testing. In addition, haplotype inference is less accurate than direct examination of the underlying causative mutations, because the defective haplotype sequence may be broken up by recombination events (VanRaden et al., 2011a). It has been recommended that valuable animals identified as carriers by haplotype analysis should be retested (VanRaden et al., 2011b; Schütz et al., 2016).

The KASP assay has been used successfully in custom genotyping of animals (e.g., Schwarzenbacher et al., 2016; Kusza et al., 2018). Unlike classic genotyping methods using Sanger sequencing or PCR-RFLP that involve several technical steps, KASP is time-effective because it requires only 1 amplification step, coupled with an end point fluorescent read of the PCR products. It takes about $2 \mathrm{~h}$ to obtain genotyping results after DNA extraction. Also, KASP shows some obvious advantages in flexibility. For example, the primers designed for this study allowed all 8 KASP assays to share the same PCR conditions, leading to improved throughput by simultaneous detection of multiple loci. Further, like the widely used TaqMan SNP genotyping method, KASP is a homogeneous and fluorescence-based assay, but its cost is reduced because there is no need to label the SNP-specific primers (He et al., 2014). In addition to the established loci, the KASP assays can easily be expanded to include new loci-another advantage over the array-based genotyping method (He et al., 2014). This is particularly important for dairy cattle because additional genetic defects, such as HH2 (VanRaden et al., 2011b; McClure et al., 2014) and HH6 (Fritz et al., 2018), will be added to the routine testing once their underlying causative mutations are uncovered.

Despite these advantages as a genotyping method based on allele-specific PCR amplification, KASP is only appropriate for small to moderate numbers of loci. For high-throughput genotyping of large numbers of genomic SNP, it is better to choose multiplexed chipbased technology.

In conclusion, KASP assays have been established and validated for rapid and easy molecular screening of 8 common genetic defects in dairy cattle. The surprisingly high frequency of the defective alleles observed in Chinese Holstein cattle warrants an immediate application of these assays for control and elimination of these genetic defects.

\section{ACKNOWLEDGMENTS}

This work was supported by the Beijing Dairy Industry Innovation Team Fund (BAIC06, China), China Agricultural Research System (CARS-37), and the Program for Changjiang Scholar and Innovation Research Team in University (IRT_15R621). We thank J. S. F. Barker for assistance in editing the English used in this article and appreciate the constructive suggestions from the anonymous peer reviewers.

\section{REFERENCES}

Adams, H. A., T. S. Sonstegard, P. M. VanRaden, D. J. Null, C. P. Van Tassell, D. M. Larkin, and H. A. Lewin. 2016. Identification of a nonsense mutation in APAF1 that is likely causal for a decrease in reproductive efficiency in Holstein dairy cattle. J. Dairy Sci. 99:6693-6701. https://doi.org/10.3168/jds.2015-10517.

Agerholm, J. S., C. Bendixen, O. Andersen, and J. Arnbjerg. 2001. Complex vertebral malformation in Holstein calves. J. Vet. Diagn. Invest. 13:283-289. https://doi.org/10.1177/104063870101300401. 
Charlier, C., J. S. Agerholm, W. Coppieters, P. Karlskov-Mortensen, W. Li, G. de Jong, C. Fasquelle, L. Karim, S. Cirera, N. Cambisano, N. Ahariz, E. Mullaart, M. Georges, and M. Fredholm. 2012. A deletion in the bovine FANCI gene compromises fertility by causing fetal death and brachyspina. PLoS One 7:e43085. https:// doi.org/10.1371/journal.pone.0043085.

Cole, J. B., D. J. Null, and P. M. VanRaden. 2016. Phenotypic and genetic effects of recessive haplotypes on yield, longevity, and fertility. J. Dairy Sci. 99:7274-7288. https://doi.org/10.3168/jds.2015 $-10777$.

Cole, J. B., P. M. VanRaden, D. J. Null, J. L. Hutchison, T. A. Cooper, and S. M. Hubbard. 2018. Haplotype tests for recessive disorders that affect fertility and other traits. AIP Research Report Genomics3. ARS-USDA, Washington, DC. https://aipl.arsusda .gov/reference/recessive_haplotypes_ARR-G3.html.

Daetwyler, H. D., A. Capitan, H. Pausch, P. Stothard, R. van Binsbergen, R. F. Brondum, X. Liao, A. Djari, S. C. Rodriguez, C. Grohs, D. Esquerre, O. Bouchez, M. N. Rossignol, C. Klopp, D. Rocha, S. Fritz, A. Eggen, P. J. Bowman, D. Coote, A. J. Chamberlain, C. Anderson, C. P. VanTassell, I. Hulsegge, M. E. Goddard, B. Guldbrandtsen, M. S. Lund, R. F. Veerkamp, D. A. Boichard, R. Fries, and B. J. Hayes. 2014. Whole-genome sequencing of 234 bulls facilitates mapping of monogenic and complex traits in cattle. Nat. Genet. 46:858-865. https://doi.org/10.1038/ng.3034.

Fang, L., Y. Li, Y. Zhang, D. Sun, L. Liu, Y. Zhang, and S. Zhang. 2013. Identification of brachyspina syndrome carriers in Chinese Holstein cattle. J. Vet. Diagn. Invest. 25:508-510. https://doi.org/ $10.1177 / 1040638713488387$.

Fritz, S., A. Capitan, A. Djari, S. C. Rodriguez, A. Barbat, A. Baur, C. Grohs, B. Weiss, M. Boussaha, D. Esquerre, C. Klopp, D. Rocha, and D. Boichard. 2013. Detection of haplotypes associated with prenatal death in dairy cattle and identification of deleterious mutations in GART, SHBG and SLC37A2. PLoS One 8:e65550. https://doi.org/10.1371/journal.pone.0065550.

Fritz, S., C. Hoze, E. Rebours, A. Barbat, M. Bizard, A. Chamberlain, C. Escouflaire, C. Vander Jagt, M. Boussaha, C. Grohs, A. Allais-Bonnet, M. Philippe, A. Vallée, Y. Amigues, B. J. Hayes, D. Boichard, and A. Capitan. 2018. An initiator codon mutation in SDE2 causes recessive embryonic lethality in Holstein cattle. J. Dairy Sci. 101:6220-6231. https://doi.org/10.3168/jds.2017-14119.

He, C., J. Holme, and J. Anthony. 2014. SNP genotyping: The KASP assay. Methods Mol. Biol. 1145:75-86.

Kipp, S., D. Segelke, S. Schierenbeck, F. Reinhardt, R. Reents, C. Wurmser, H. Pausch, R. Fries, G. Thaller, and J. Tetens. 2015. A new Holstein haplotype affecting calf survival. Interbull Bull. 49:49-53.

Kusza, S., L. T. Cziszter, D. E. Ilie, M. Sauer, I. Padeanu, and D. Gavojdian. 2018. Kompetitive Allele Specific PCR (KASP) genotyping of 48 polymorphisms at different caprine loci in French Alpine and Saanen goat breeds and their association with milk composition. PeerJ 6:e4416. https://doi.org/10.7717/peerj.4416.

McClure, M. C., D. Bickhart, D. Null, P. Vanraden, L. Xu, G. Wiggans, G. Liu, S. Schroeder, J. Glasscock, J. Armstrong, J. B. Cole,
C. P. Van Tassell, and T. S. Sonstegard. 2014. Bovine exome sequence analysis and targeted SNP genotyping of recessive fertility defects $\mathrm{BH} 1, \mathrm{HH} 2$, and $\mathrm{HH} 3$ reveal a putative causative mutation in SMC2 for HH3. PLoS One 9:e92769. https://doi.org/10.1371/ journal.pone.0092769.

Menzi, F., N. Besuchet-Schmutz, M. Fragniere, S. Hofstetter, V. Jagannathan, T. Mock, A. Raemy, E. Studer, K. Mehinagic, N. Regenscheit, M. Meylan, F. Schmitz-Hsu, and C. Drogemuller. 2016. A transposable element insertion in APOB causes cholesterol deficiency in Holstein cattle. Anim. Genet. 47:253-257. https://doi .org/10.1111/age.12410.

Schütz, E., C. Wehrhahn, M. Wanjek, R. Bortfeld, W. E. Wemheuer, J. Beck, and B. Brenig. 2016. The Holstein Friesian lethal haplotype 5 (HH5) results from a complete deletion of TBF1M and cholesterol deficiency (CDH) from an ERV-(LTR) insertion into the coding region of APOB. PLoS One 11:e0154602. https://doi .org/10.1371/journal.pone.0154602.

Schwarzenbacher, H., C. Wurmser, K. Flisikowski, L. Misurova, S. Jung, M. C. Langenmayer, A. Schnieke, G. Knubben-Schweizer, R. Fries, and H. Pausch. 2016. A frameshift mutation in GON4L is associated with proportionate dwarfism in Fleckvieh cattle. Genet. Sel. Evol. 48:25. https://doi.org/10.1186/s12711-016-0207-z.

Segelke, D., H. Täubert, F. Reinhardt, and G. Thaller. 2016. Considering genetic characteristics in German Holstein breeding programs. J. Dairy Sci. 99:458-467. https://doi.org/10.3168/jds.2015-9764.

Semagn, K., R. Babu, S. Hearne, and M. Olsen. 2014. Single nucleotide polymorphism genotyping using Kompetitive Allele Specific PCR (KASP): Overview of the technology and its application in crop improvement. Mol. Breed. 33:1-14. https://doi.org/10.1007/ s11032-013-9917-x.

Shuster, D. E., J. M. E. Kehrli, M. R. Ackermann, and R. O. Gilbert. 1992. Identification and prevalence of a genetic defect that causes leukocyte adhesion deficiency in Holstein cattle. Proc. Natl. Acad. Sci. USA 89:9225-9229.

Thomsen, B., P. Horn, F. Panitz, E. Bendixen, A. H. Petersen, L. E. Holm, V. H. Nielsen, J. S. Agerholm, J. Arnbjerg, and C. Bendixen. 2006. A missense mutation in the bovine SLC35A3 gene, encoding a UDP-N-acetylglucosamine transporter, causes complex vertebral malformation. Genome Res. 16:97-105. https://doi.org/ 10.1101/gr.3690506.

VanRaden, P. M., D. J. Null, K. M. Olson, and J. L. Hutchison. 2011a. Reporting of haplotypes with recessive effects on fertility. Interbull Bull. 44:117-121. https://journal.interbull.org/index.php/ib/ article/view/1202/1287.

VanRaden, P. M.. K. M. Olson, D. J. Null, and J. L. Hutchison. 2011b. Harmful recessive effects on fertility detected by absence of homozygous haplotypes. J. Dairy Sci. 94:6153-6161. https://doi.org/10 $.3168 /$ jds.2011-4624.

Zhang, Y., X. Fan, D. Sun, Y. Wang, Y. Yu, Y. Xie, S. Zhang, and Y. Zhang. 2012. A novel method for rapid and reliable detection of complex vertebral malformation and bovine leukocyte adhesion deficiency in Holstein cattle. J. Anim. Sci. Biotechnol. 3:24. https: //doi.org/10.1186/2049-1891-3-24. 